\title{
441.
}

\section{NOTE SUR L'ALGORITHME DES TANGENTES DOUBLES D'UNE COURBE DU QUATRIEME ORDRE.}

[From the Journal für die reine und angewandte Mathematik (Crelle), tom. LxvIII. (1868), pp. $176-179$.]

ON n'a pas, je crois, assez fait attention à l'algorithme (tiré de la considération d'une figure dans l'espace) qu'a trouvé M. Hesse (dans le mémoire "Ueber die Doppeltangenten der Curven vierter Ordnung," t. XLIX de ce Journal, 1855) pour dénoter les tangentes doubles (ou bitangentes) d'une courbe du quatrième ordre. Voici en quoi cet algorithme consiste. En employant les huit symboles 1, 2, 3, . 8, les 28 bitangentes sont représentées par les combinaisons binaires 12, 13, 14, ..78. Cela posé, considérons une expression quelconque 12.13.14, ou $12.34, \ldots$ ou disons un "terme" qui représente un système d'une seule ou de plusieurs des bitangentes. On peut opérer sur ce terme avec deux espèces de substitutions; la substitution ordinaire qui consiste à changer l'arrangement 12345678 des huit symboles en un autre arrangement quelconque; et la substitution "bifide" représentée par un symbole tel que 1234.5678, lequel dénote qu'il faut entrechanger les combinaisons 12 et 34,13 et 24,14 et 23,56 et 78 , 57 et 68 , 58 et 67 , en ne changeant pas les autres combinaisons. Par exemple en opérant avec 1234.5678 sur 34.45 .56 .17 on obtient 12.45.78.17. Le nombre de ces substitutions bifides est 35 , ou en comptant la substitution, unité, qui ne change aucune des combinaisons, ce nombre est 36 .

Appelons "homotypiques" deux termes qui se dérivent l'un de l'autre par une substitutioń ordinaire; "syntypiques" qui se dérivent l'un de l'autre par une substitution ordinaire ou bifide; "sous-groupe" le système entier des termes homotypiques à un terme donné: "groupe" le système entier des termes syntypiques à un terme donné. Un groupe peut contenir un seul sous-groupe, ou plusieurs sous-groupes; mais il importe de remarquer que la notion du sous-groupe n'a pas de signification géométrique, et ne $16-2$ 
sert que comme moyen de former les termes du groupe. Cela étant, le théorème géométrique est celui-ci; "les systèmes de bitangentes représentées par des termes syntypiques (ou autrement dit, par des termes qui appartiennent au même groupe) ont les mêmes propriétés géométriques."

Par exemple, en considérant les bitangentes deux à deux, on a deux sous-groupes, l'un composé de termes homotypiques à 12.13; l'autre, de termes homotypiques à 12.34 - ou disons, le sous-groupe 12.13 de 168 termes et le sous-groupe 12.34 de 210 termes; mais ces deux sous-groupes ne forment qu'un seul groupe: pour montrer cela il suffit d'opérer sur 12.13, par exemple avec la substitution 1245.3678 , ce qui donne 45.13 , terme homotypique à 12.34 . Cela veut dire qu'il n'y a pas de combinaison de deux bitangentes qui se distingue d'une manière quelconque de toute autre combinaison de deux bitangentes.

Mais en combinant les bitangentes trois à trois, on a les deux sous-groupes 12.34 .56 (420 termes) et 12.23.34 (840 termes) qui forment un groupe de 1260 termes; les trois bitangentes représentées par un quelconque des 1260 termes ont leurs six points de contact sur une même conique. Les trois autres sous-groupes 12.23.31 (56 termes), 12.23.45 (1680 termes) et 12.13.14 (280 termes) forment un groupe de 2016 termes, et pour trois bitangentes représentées par un terme quelconque de ce groupe, les six points de contact ne sont pas situés sur une même conique.

Comme un autre exemple j'explique la constitution des 63 "groupes" de Steiner (voir le mémoire de Steiner, "Eigenschaften der Curven vierten Grades rücksichtlich ihrer Doppeltangenten," t. xuIx. de ce journal, 1855) ou (pour éviter l'emploi de ce mot groupe dans une nouvelle signification) disons les 63 termes $G$ de Steiner, chaque terme composé de 6 paires de bitangentes. On a ici un sous-groupe de 35 termes $G_{1}$ de la forme

$$
12.34 ; 13.42 ; 14.23 ; 56.78 ; 57.86 ; 58.67
$$

(pour abréger on peut dénoter ce terme par 1234.5678), et un sous-groupe de 28 termes $G_{2}$ de la forme

$$
13.32 ; 14.42 ; 15.52 ; 16.62 ; 17.72 ; 18.82
$$

(pour abréger on peut de même dénoter ce terme par 12.345678), les deux sousgroupes forment le groupe des 63 termes $G$.

Steiner a de plus considéré les "systèmes" ou disons les termes $S_{1}, S_{2}$, composés chacun de trois termes $G$; savoir 315 termes $S_{1}$ et 336 termes $S_{2}$. Les 315 termes $S_{1}$ sont ici un groupe composé d'un sous-groupe de 105 termes $3 G_{1}$ de la forme

$$
1234.5678 ; 1256.3478 ; 1278.3456
$$

et un sous-groupe de 210 termes $2 G_{2}+G_{1}$ de la forme

$$
12.345678 ; 34.125678 \text { et } 1234.5678 \text {. }
$$

Et de même les $3: 36$ termes $S_{2}$ sont un groupe composé d'un sous-groupe de 280 termes $2 G_{1}+G_{2}$ de la forme

$$
1234.5678 ; 5234.1678 \text { et } 15.234678
$$


et un sous-groupe de 56 termes $3 G_{2}$ de la forme

\section{$12.345678 ; 13.24 .5678 ; 31.245678$.}

Il va sans dire que je me suis servi de l'abréviation 1234.5678 pour dénoter le terme $12.34 ; 13.42 ; 14.23 ; 56.78 ; 57.86 ; 58.67$; et de même pour les autres termes $G_{1}$ ou $G_{2}$.

M. Aronhold (dans le mémoire "Ueber den gegenseitigen Zusammenhang der 28 Doppeltangenten einer allgemeinen Curve vierten Grades," Berl. Monatsber. Juli 1864), partant de 7 bitangentes données, a trouvé une construction pour les autres 21 bitangentes. Les bitangentes données doivent être indépendantes; savoir pour trois quelconques de ces 7 bitangentes, les six points de contact ne sont pas situés sur une même conique. Les bitangentes représentées par les termes 12, 13, 14, 15, 16, 17, 18 sont un tel système de bitangentes indépendantes; et en dénotant de cette manière les 7 bitangentes données, la bitangente construite par le moyen de la conique qui touche cinq de ces droites, par exemple les droites $38,48,58,68,78$, (ou conique 34567 ) peut être dénotée par 12, et de même pour les autres bitangentes cherchées; on a ainsi le système entier des bitangentes dénotées comme auparavant par $12,13,14, \ldots 78$.

J'ajoute que le groupe qui contient $18,28,38,48,58,68,78$ est composé d'un sousgroupe $18,28,38,48,58,68,78$ de 8 termes, et d'un sous-groupe $12,23,31,48,58,68,78$ de 280 termes; le groupe contient donc 288 termes; savoir il y a ce nombre 288 de systèmes de sept bitangentes indépendantes qui peuvent chacun servir à trouver par la construction d'Aronhold les autres 21 bitangentes.

P.S. J'ai trouvé à propos de la méthode de M. Aronhold une forme commode pour l'équation de la conique qui touche cinq droites données; en supposant que l'on ait identiquement $x+y+z+w=0$, et que les droites données soient $x=0, y=0, z=0, w=0$, et $a x+b y+c z+d w=0$, l'équation de la conique est

$$
(a-d)^{2}(b-c)^{2}(x w+y z)+(b-d)^{2}(c-a)^{2}(y w+z x)+(c-d)^{2}(a-b)^{2}(z w+x y)=0 .
$$

J'ajoute qu'en écrivant pour abréger

$$
\alpha: \beta: \gamma=(a-d)(b-c):(b-d)(c-a):(c-d)(a-b)
$$

(d'où $\alpha+\beta+\gamma=0$ ) les coordonnées $(x, y, z, w)$ des points de contact avec les droites

$$
x=0, \quad y=0, \quad z=0, \quad w=0 \text { sont }(0, \gamma, \beta, \alpha),(\gamma, 0, \alpha, \beta),(\beta, \alpha, 0, \gamma),(\alpha, \beta, \gamma, 0)
$$

respectivement; et que les coordonnées du point de contact avec la droite $a x+b y+c z+d w=0$ sont

$$
x: y: z: w=(b c d):-(c d a):(d a b):-(a b c)
$$

où, pour abréger, $(b c d)$ dénote $(b-c)(c-d)(d-b)$, et de même pour $(c d a),(d a b),(a b c)$.

Cambridge, le 23 septembre 1867. 